\section{Androgen-Suppression: Sechs Monate effektiv?}

\begin{abstract}
Bei lokal fortgeschrittenem Prostatakarzinom verlängert die langfristige Androgen-Suppression das Überleben. Ob sechs Monate ebenso wirksam sind wie drei Jahre, hat ein europäisches Autorenteam untersucht.
\end{abstract}

\begin{abstract}
A n der Studie der EORTC Radiation Oncology Group und der GenitoUrinary Tract Cancer Group nahmen 970 Männer mit Prostatakarzinomen der Stadien T1c-T2b, N1-N2 ohne Fernmetastasen teil. Alle erhielten eine externe Radiotherapie mit einer Drei-oder Vierfelder-Bestrahlung. Dabei wurde das gesamte Becken mit 50 Gy, danach nochmals Prostata und die Vesiculae seminales mit 20 Gy bestrahlt.

Randomisiert erhielten danach 483 Patienten eine kurzzeitige Androgen-Supression für nur sechs Monate, 487 unterzogen sich einer Langzeit-Suppression
\end{abstract}

für insgesamt drei Jahre. In den ersten sechs Monaten wurde in beiden Gruppen vom ersten Bestrahlungstag an eine komplette Androgenblockade mit einem LHRH-Agonisten und einem Antiandrogen (750 mg Flutamid oder $50 \mathrm{mg}$ Bicalutamid pro Tag) durchgeführt. Die Behandlung wurde in der ersten Gruppe dann beendet, die Patienten in der zweiten Gruppe erhielten das LHRH-Analogon für weitere zweieinhalb Jahre.

Nach median 6,4 Jahren waren 132 Patienten der ersten Gruppe und $98 \mathrm{~Pa}$ tienten aus der zweiten Gruppe verstorben. Die 5-Jahres-Gesamtmortalitätsrate lag bei 19,0 bzw. 15,2\%. Im Test auf Nichtunterlegenheit war die KurzzeitSuppression damit unterlegen.

Unter Kurzzeit-Suppression traten 47, unter Langzeit-Suppression 29 tumorbedingte Todesfälle auf. Die Karzinom-spezifischen Mortalitätsraten über fünf Jahre betrugen $4,7 \%$ in der ersten und $3,2 \%$ in der zweiten Gruppe, dieser Unterschied war mit $\mathrm{p}=0,002$ signifikant.

Fazit: Beim lokal fortgeschrittenen Prostatakarzinom erwies sich in dieser Studie die kurzzeitige Androgen-Suppression von sechs Monaten gegenüber einer Langzeitbehandlung von drei Jahren als unterlegen.

Bolla $M$ et al. Duration of androgen suppression in the treatment of prostate cancer. New Engl J Med 2009; 360: 2516-27.

\section{MDM2 und Ki-67 als Prognosemarker}

\section{Das Onkoprotein MDM2, das eine Deregulation des Tumorsuppressor-} proteins $\mathrm{p} 53$ bewirkt, ist bei vielen Tumoren überexprimiert. Erste Hinweise zeigen, dass sich beim Prostatakarzinom MDM2 wie auch der Proliferationsmarker Ki-67 als Prognosemarker eignen könnten.

B eim Prostatakarzinom wurde einerseits ein Zusammenhang der Überexpression von MDM2 mit dem Gleason-Score beobachtet. Unabhängig davon zeigte sich im Rahmen einer randomisierten Phase-III-Studie bei Männern mit Prostatakarzinom, dass Ki-67 und p53 sowohl in Bezug auf Fernmetastasen als auch auf die krankheitsspezifische Mortalität als Prädiktoren infrage kommen. Bei einem Teil der Patienten dieser Studie, die eine Radiotherapie plus Andro-

gendeprivation entweder über einen kurzen oder einen langen Zeitraum erhalten hatten, wurde nun überprüft, ob sich die veränderte Expression dieser Biomarker tatsächlich zur Einschätzung der Progression eignet.

In Gewebeproben von insgesamt 478 Patienten konnte die Expression von MDM2, Ki-67 und p53 quantitativ bestimmt werden. Eine MultivarianzAnalyse der bereinigten Daten zeigte, dass eine Überexpression von MDM2

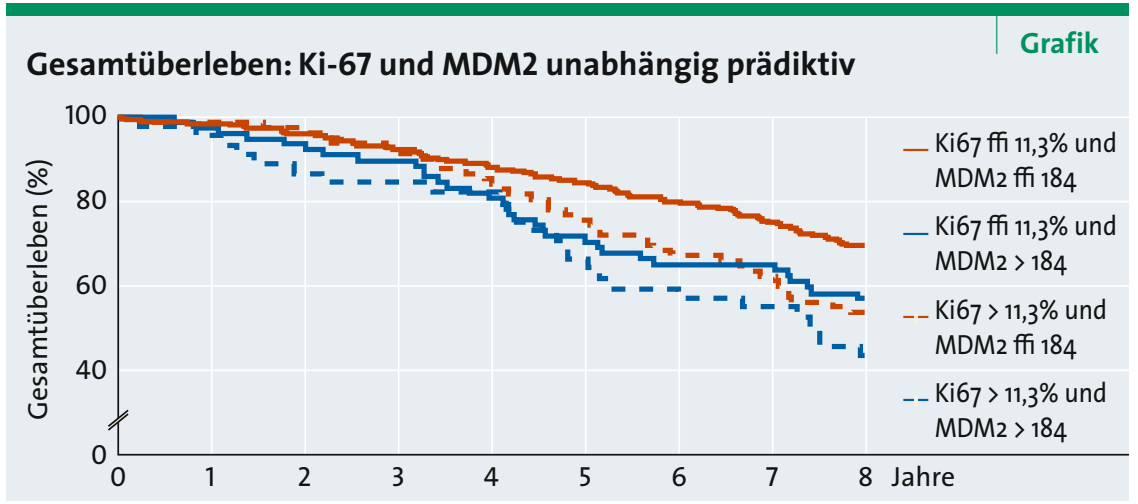

deutlich mit dem Auftreten von Fernmetastasen $(\mathrm{p}=0,02)$ und der Gesamtmortalität $(\mathrm{p}=0,003)$ korrelierte. Ebenfalls gab es einen Zusammenhang zwischen einer überhöhten Ki-67-Expression und dem Auftreten von Fernmetastasen ( $\mathrm{p}<$ $0,0001)$ bzw. der Gesamtmortalität ( $\mathrm{p}=$ $0,0007)$. Außerdem zeigte sich hier eine Korrelation mit der ursachenspezifischen Mortalität $(p=0,01)$. In Bezug auf p53 korrlierten hingegen nur Überexpression und Gesamtmortalität $(\mathrm{p}=0,02)$. Waren sowohl Ki-67 als auch MDM2 überexprimiert, war das Risiko für Fernmetastasen sowie Tod - krankheitsbedingt oder nicht - erhöht (jeweils $\mathrm{p}<0,001$ ).

Fazit: Beim Prostatakarzinom scheint die kombinierte Überexpression von MDM2 und Ki-67 deutlich mit dem Auftreten von Fernmetastasen sowie der Mortalität in Zusammenhang zu stehen. Bestätigt sich dies in weiteren Studien, so könnten diese Parameter aus Sicht der Autoren Marker für das Patienten-Outcome abgeben sowie zur Risikostratifikation in klinischen Studien taugen. aro

Khor $\mathrm{LY}$ et al. MDM2 and Ki-67 predict for distant metastasis and mortality in men treated with radiotherapy and androgen deprivation for prostate cancer: RTOG 92O2. J Clin Oncol 2009; 27: 3177-84. 